\title{
Effects of Online Augmented Kinematic and Perceptual Feedback on Treatment of Speech Movements in Apraxia of Speech
}

\author{
M.R. McNeil ${ }^{a, b}$ W.F. Katz ${ }^{c, d}$ T.R.D. Fossett ${ }^{a, b}$ D.M. Garst ${ }^{c, d}$ N.J. Szuminsky ${ }^{a, b}$ \\ G. Carter ${ }^{d}$ K.Y. Lim ${ }^{a, b}$ \\ ${ }^{a}$ Geriatric Research Education and Clinical Center, VA Pittsburgh Healthcare System, and b University of Pittsburgh, \\ Pittsburgh, Pa., 'Callier Center for Communication Disorders, University of Texas at Dallas, and d Dallas Veterans \\ Administration Medical Center, Dallas, Tex., USA
}

\section{Key Words}

Apraxia of speech • Augmented feedback • Kinematic

feedback • Perceptual feedback

\begin{abstract}
Apraxia of speech (AOS) is a motor speech disorder characterized by disturbed spatial and temporal parameters of movement. Research on motor learning suggests that augmented feedback may provide a beneficial effect for training movement. This study examined the effects of the presence and frequency of online augmented visual kinematic feedback (AVKF) and clinician-provided perceptual feedback on speech accuracy in 2 adults with acquired AOS. Within a single-subject multiple-baseline design, AVKF was provided using electromagnetic midsagittal articulography (EMA) in 2 feedback conditions (50 or 100\%). Articulator placement was specified for speech motor targets (SMTs). Treated and baselined SMTs were in the initial or final position of single-syllable words, in varying consonant-vowel or vowel-consonant contexts. SMTs were selected based on each participant's pre-assessed erred productions. Productions were digitally recorded and online perceptual judgments of accuracy (including segment and intersegment distortions) were made. Inter- and intra-judge reliability for perceptual accuracy was
\end{abstract}

high. Results measured by visual inspection and effect size revealed positive acquisition and generalization effects for both participants. Generalization occurred across vowel contexts and to untreated probes. Results of the frequency manipulation were confounded by presentation order. Maintenance of learned and generalized effects were demonstrated for 1 participant. These data provide support for the role of augmented feedback in treating speech movements that result in perceptually accurate speech production. Future investigations will explore the independent contributions of each feedback type (i.e. kinematic and perceptual) in producing efficient and effective training of SMTs in persons with AOS.

Copyright $\odot 2010$ S. Karger AG, Basel

\section{Introduction}

Apraxia of speech (AOS) is a motor speech disorder characterized by disrupted motor planning [1] and/or motor programming $[2,3]$. Disturbed components of the motor planning to programming continuum include the spatial and temporal parameters of movement. These disturbed components are responsible for the core behaviors that define AOS, including sound distortions, lengthened

\section{KARGER}

Fax +41613061234

E-Mail karger@karger.ch

www.karger.com (c) 2010 S. Karger AG, Basel

Accessible online at: www.karger.com/fpl
Prof. Malcolm R. McNeil

Department of Communication Science and Disorders, University of Pittsburgh 4033 Forbes Tower

Pittsburgh, PA 15260 (USA)

Tel. +1 412383 6541, Fax +1 412383 6555, E-Mail mcneil@ pitt.edu 
segments, lengthened intersegment durations and disturbed prosody [2]. Based on these mechanisms, it is logical to hypothesize that principles of motor learning (i.e. practice, role of feedback) may be applicable to the treatment of AOS [2, 4]. Wambaugh et al. [5] reviewed the AOS treatment literature and concluded that some form of feedback is typically provided in AOS treatment studies. About half of the existing AOS treatment studies have focused on the spatial and temporal parameters of movement [5].

Augmented feedback is that which provides information about movement outcome and typically occurs along with inherent or intrinsic feedback. Knowledge of results is one type of augmented feedback that provides information about the outcome of the target movement when the individual has knowledge about the movement goal before its initiation [6]. Knowledge of performance is augmented feedback that provides information about the movement pattern. Feedback frequency is one dimension of augmented feedback that can affect the acquisition, transfer and maintenance of learning $[2,7,8]$, but there are contrasting data [9]. Acquisition is defined as learning that occurs during practice, while retention reflects maintenance or learning over time, without the augmented feedback. Transfer or generalization is learning of an untrained behavior [5]. Some AOS treatment studies have explicitly incorporated motor learning principles in treatment $[10,11]$. Though overall positive treatment effects have been demonstrated in AOS treatment studies targeting speech movements, a recent evaluation of the literature concluded that there is a critical need for more efficacy and effectiveness data $[6,12]$.

The current study sought to determine if the presence and/or frequency of online, concurrent and terminal augmented visual kinematic feedback (AVKF), plus online clinician-provided perceptual feedback, would lead to perceptually judged improved accuracy for selected SMTs. Hypotheses were that: (1) perceptually judged SMT accuracy would improve with treatment compared to baseline conditions, (2) generalization of learned SMTs would occur to untreated SMTs and both treated and untreated gains would be maintained with the removal of treatment, and (3) 100\% concurrent feedback frequency would provide greater acquisition compared to the $50 \%$, but that maintenance and generalization of acquired SMTs to other speech contexts would be greater for those trained under $50 \%$ feedback. Visual inspection of the graphed data and effect sizes were calculated across baseline, acquisition, generalization and maintenance phases to determine treatment effects.

\section{Methods and Procedures}

\section{Participants}

Diagnosis of AOS was made by the first author and confirmed by non-independent collaborators, based on repeated productions of single-syllable words as well as productions elicited from other subtests of the Apraxia Battery for Adults-2 [13] and connected speech. Criteria for diagnosis included slow speaking rate, sound distortions, distorted sound substitutions, extended segments and/or intersegment durations, and disturbed prosody. Several cognitive-linguistic and speech motor descriptive and screening measures were administered to further characterize participant's speech and motor skills. Graphs for all baselined, treated and maintenance data are available from the first author upon request.

Participants were 2 adults with AOS and concomitant aphasia. Participant 1 (P1) was a 45-year-old, right-handed female, presenting 26 months after onset of a large left-hemisphere thromboembolic stroke involving the left anterolateral frontal lobe and insular cortex. P1 showed mild-moderate aphasia, characterized by phonological paraphasias and mild-moderate AOS. Scores on the word, command and complex ideas auditory comprehension subtests from the Boston Diagnostic Aphasia Exam [14] were 94, 40 and $33 \%$, respectively. She scored in the 39th percentile on the Computerized Revised Token Test (a measure of auditory comprehension; unpublished), 100\% (36/36) on the Raven's Colored Progressive Matrices [15] and produced 19\% information units per min in the Story Retell Procedure [16]. P1 had previously received speech-language treatment that focused on phonological speech sound production with undocumented improvement reported prior to study enrollment.

Participant 2 (P2) was a 38-year-old male, presenting 2 years after onset of a left intraparenchymal hemorrhagic stroke involving the left frontal/temporal cortical region with extension into the basal ganglia. He was diagnosed with mild-moderate aphasia and severe AOS. Scores on the word, command and complex ideas auditory comprehension subtests from the Boston Diagnostic Aphasia Exam [14] were 93, 90 and 17\%, respectively. He scored in the 26th percentile on the Computerized Revised Token Test (unpublished); scored 30/36 on the Raven's Colored Progressive Matrices [15] and 0\% information units per min on the Story Retell Procedure [16] because his speech was unintelligible. He had received no speech-language treatment prior to study enrollment. P2's speech production was characterized most prominently by disturbed prosody, lengthened segments and sound distortions. His language production was limited and characterized by substantive difficulties with word retrieval. P2 was discontinued from the study for health issues unrelated to the study following treatment of the second SMT.

\section{Treatment Targets and Non-Treated Probes}

Based on each participant's unique pattern of errors elicited on a battery of words with varied phonemic (consonant-vowel) constructions, 4 SMTs were selected for each participant. Subsequently, at least 3 baseline pre-treatment probes were administered. SMTs were constrained by ease of visualization of tongue movement with electromagnetic midsagittal articulography (EMA) and occurred in the initial or final position of 1-syllable words. Each SMT occurred within 5 different vowel contexts for P1 and were represented by 4 different sets of consonants $(/ d /$, 
$/ \mathrm{t} / / \mathrm{g} /$ and $/ \mathrm{s} /)$, and 2 different sets of consonants $(/ \mathrm{g} /$ and $/ \mathrm{n} /)$ for P2. This yielded a total of 20 treatment stimuli for P1 and 10 for P2. Each SMT was accompanied by 5 additional non-treated stimuli containing the same initial SMT in additional vowel contexts as well as several unrelated SMTs that served as control stimuli and were used to measure generalization and maintenance. $\mathrm{P} 1$ and $\mathrm{P} 2$ received 60 and 70 non-treated probe stimuli, respectively.

Treated stimuli yielded approximately 200 correct productions (40 correct productions for each consonant-vowel or vowelconsonant SMT context) during each treatment/practice session. During treatment, criterion for production accuracy required both kinematic and clinician-judged perceptual accuracy. Treated stimuli were regularly probed throughout the course of treatment at the beginning of each session and untreated generalization targets were periodically probed before practice on the treatment stimuli. A criterion of $80 \%$ (4 of 5 treatment STMs) or higher accuracy (judged perceptually) was required before treatment for that set was discontinued and treatment for another set of SMTs was initiated.

\section{Procedures}

All procedures were conducted in a sound-treated IAC booth. Participants were seated in a chair facing a computer monitor. EMA technology was used to deliver the online AVKF. Both AVKF and clinician-provided feedback were visually displayed on the monitor by a defined target that changed colors with accurate tongue placement and by lighted yes/no buttons indicating clinician-judged perceptual accuracy. Participants were fitted with the EMA helmet that produced a triangulated magnetic field within which calibrated sensors recorded and displayed tongue movements. The sensors were glued midline to participants' tongue tip (approximately $6 \mathrm{~mm}$ from the tip), tongue dorsum and lower lip. Sensor traces showing tongue tip position and movement provided the visual feedback on the computer monitor along with the predetermined circular target zone. A custom program provided the feedback. Before daily treatment commenced, a few accurate initial attempts at the SMT were elicited to determine the target region for accurate sound and movement productions. AVKF provided by EMA, consisted of knowledge of performance from the visible movement trace. Additionally, knowledge of results was provided by the examiner's online perceptual judgments. SMTs were randomly selected for 50 or $100 \%$ feedback conditions. Long-term maintenance was obtained 1 month after treatment termination for P1 and was not obtained for P2 because of early study termination.

\section{Data Design and Analysis}

Data were collected within a single-subject multiple baseline/ across behaviors experimental design. Data were analyzed with both visual inspection and effect size calculations. Inter- and intra-judge reliability was obtained for online perceptual judgments, across multiple sounds and sessions for 1 participant. Visual inspection was judged by 2 experienced judges for variability, magnitude and slope of data across study phases. Both judgments and effect sizes were computed across true baseline to: (1) treatment, (2) post-treatment, (3) untreated generalization, and (4) maintenance.

Augmented Kinematic and Acoustic

Feedback Treatment in AOS

\section{Results}

\section{Reliability}

Intra-judge reliability for the visual inspection analyses for 3 SMTs averaged $88 \%$ for one judge and $95 \%$ for the other, and inter-judge reliability averaged $94 \%$ for those same data.

\section{Acquisition, Generalization and Maintenance}

A small subset of representative data for both participants is presented in figure 1 (a complete set of figures for the treated and untreated data can be obtained from the first author). Vertical dotted lines represent separate phases of the treatment regimen. The treated (shaded areas) or pre- or post-treatment baselined words within which the SMTs were encompassed are labeled at the top of each panel. Numbers under each panel represent the session number. Values to the left of each plot represent percent accuracy. Panel 1 for P1 illustrates the 1st treated SMT (represented by the word 'duck'). While some baseline variability is evident, improved accuracy following AVKF was immediate and remained consistently high during treatment and during the treatment of the SMTs organized around / $\mathrm{t} / \mathrm{initial}$ stimuli. There was a drop to baseline during treatment of the SMTs with sound initial $\mathrm{g} / \mathrm{and}$ a return during /s/ SMTs and at maintenance. A similar pattern was evidence for the /t $\mathrm{f} /$ initial, /g/ initial and /t $\mathrm{t} /$ final SMTs. Stable baselines without generalization on the subsequent and sequentially treated SMTs provide the experimental control with which to attribute the change during the treatment to the intervention. Evidence for generalization is provided by the improvement in the untreated /g/ initial SMT within the word 'gown' during the treatment of other /g/ SMTs. Maintenance of improvements was seen for all of the targets displayed except for that of the /s/ initial ('soy') SMT, which yielded no generalization effects and provides additional evidence of experimental control for the treated SMTs.

A positive treatment effect was judged from visual inspection for 5 of the $5 / d /$ initial SMTs (4 met criterion; overall effect size, ES $=1.05)$, on 5 of the $5 / \mathrm{t} / \mathrm{initial} \mathrm{SMTs}$ ( 3 met criterion; ES $=2.06$ ), on 5 of the $5 / \mathrm{g} /$ initial SMTs ( 4 met criterion; ES $=7.17$ ) and on 5 of the $5 / \mathrm{s} /$ initial SMTs (4 met criterion; ES $=1.73$ ). Generalization of treatment to untreated controls sharing the same sound initial SMT yielded 4 of 5 positive effects $(2$ met criterion; ES = 0.45 ) for the /d/ initial SMTs, 5 of the $5 / \mathrm{t} / /$ initial SMTs ( 2 met criterion; ES $=2.6$ ), 5 of the $5 / \mathrm{g} /$ initial SMTs ( 3 met criterion; ES $=6.08$ ) and 2 of the $5 / \mathrm{s} /$ initial SMTs (1 met criterion; ES = 1.05). None of the $10 / \mathrm{t} / / \mathrm{SMTs}$ dem- 


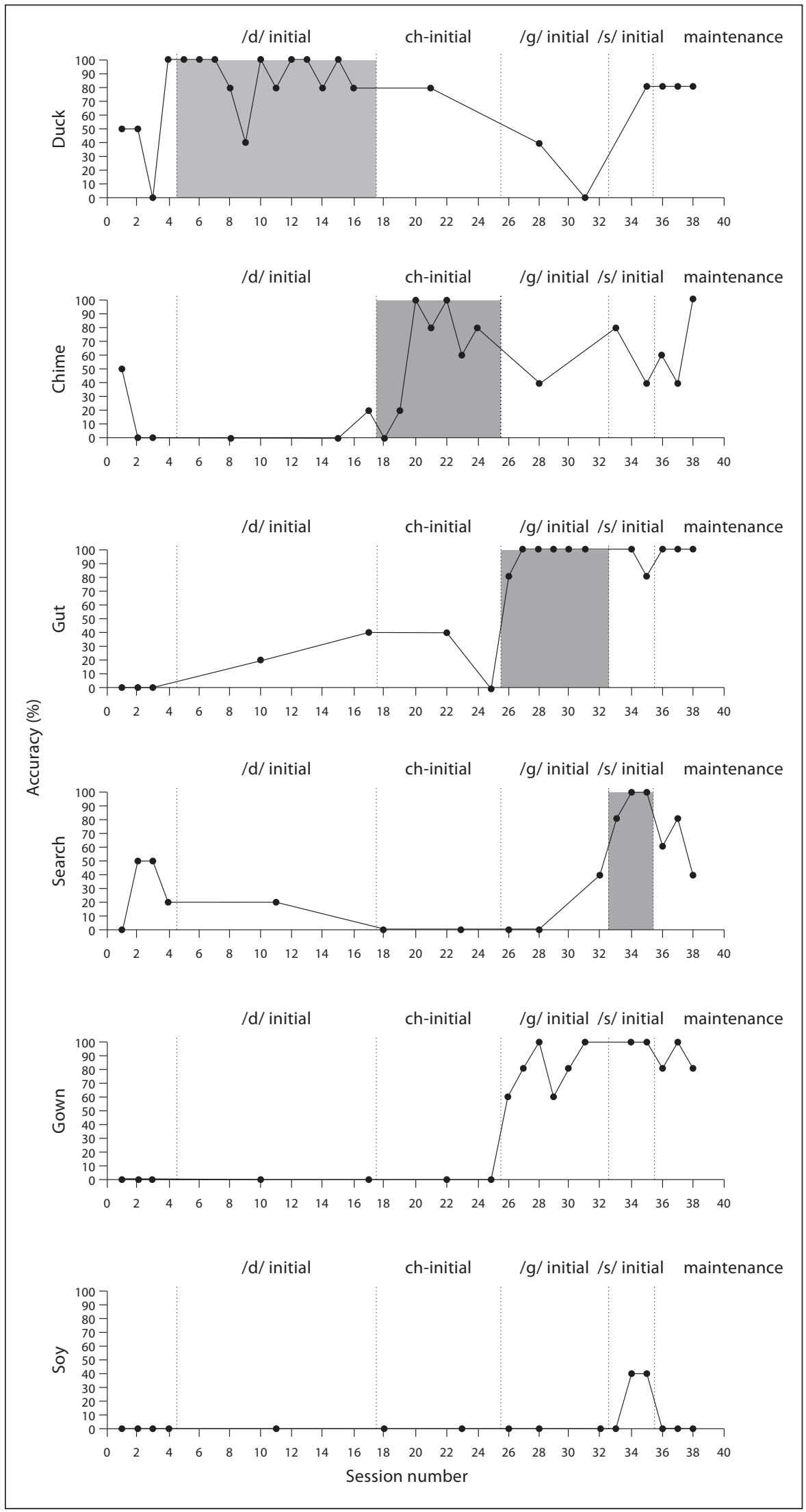
pant 1. 
Fig. 1. Representative data for participant 2 .

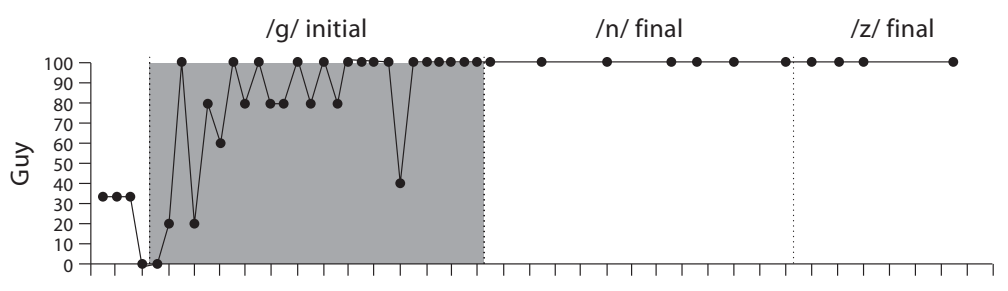

$02246 \quad 810121416182022242628303234363840424446485052545658606264666870$

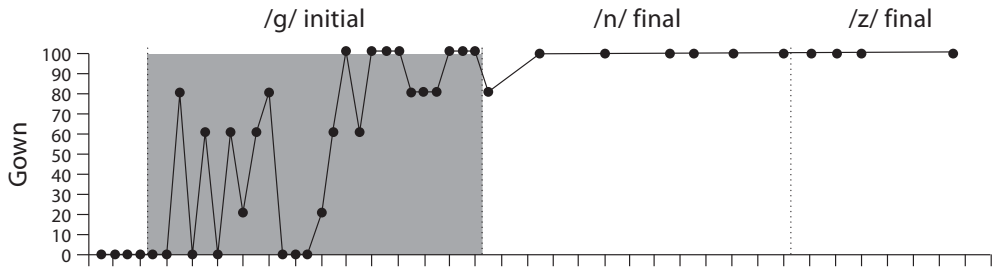

$0 \quad 246 \quad 8 \quad 10121416182022242628303234363840424446485052545658606264666870$

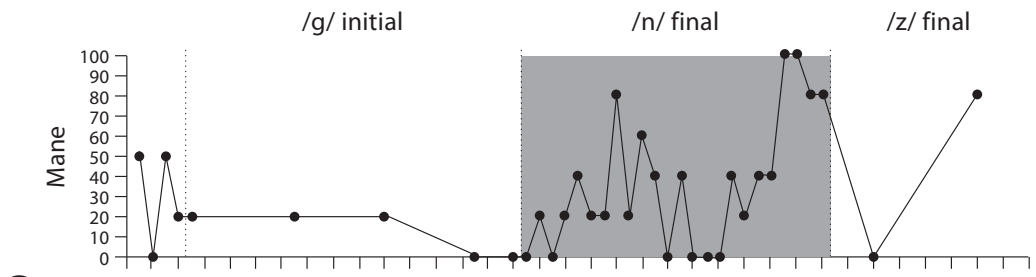

ఏ $\quad 02446810121416182022242628303234363840424446485052545658606264666870$

ป⿱艹

/g/initial

/n/ final /z/ final
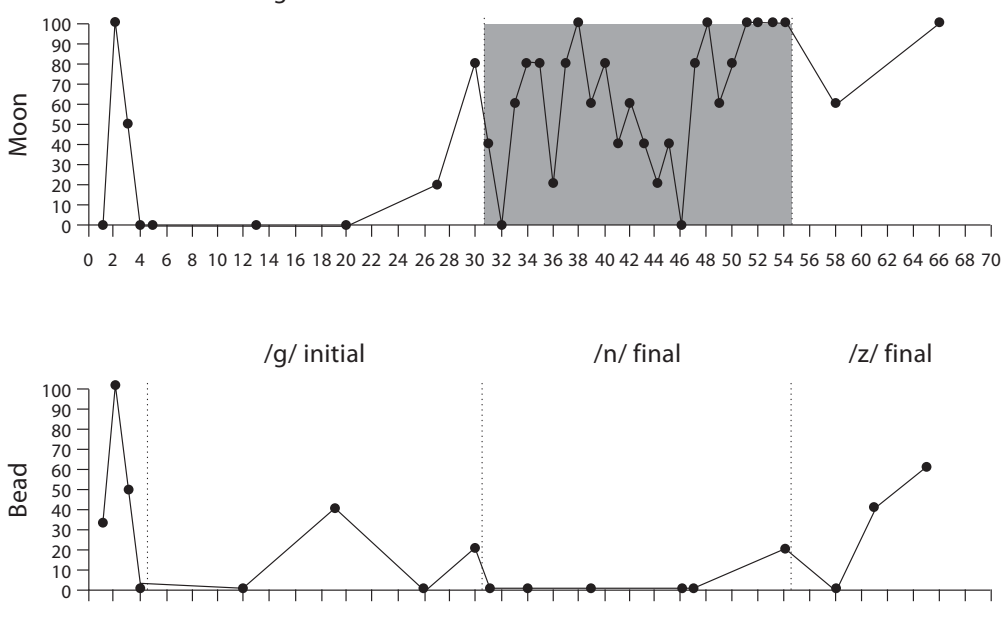

$02246 \quad 810121416182022242628303234363840424446485052545658606264666870$

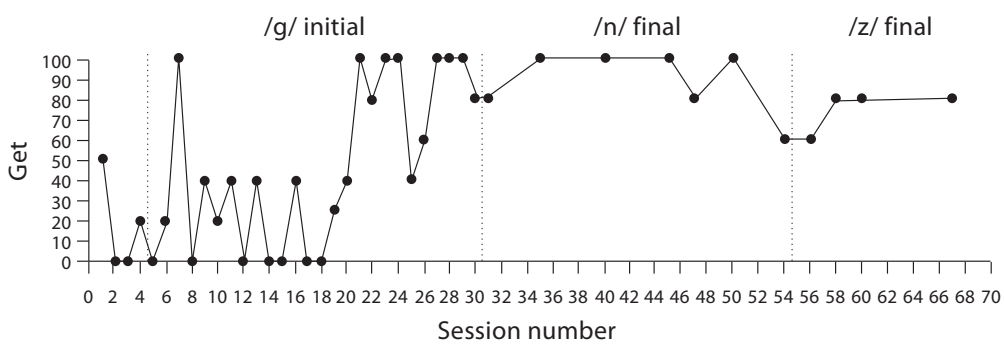


onstrated generalization during treatment of $/ \mathrm{d} / ; 6$ of 10 $/ \mathrm{g} / \mathrm{SMTs}$ demonstrated generalization during treatment of $/ \mathrm{d} / \mathrm{SMTs}(\mathrm{ES}$ treated $=2.24$; ES untreated $=2.77), 2$ of 10 /g/ SMTs evidenced generalization during /t $/$ SMT treatment $(\mathrm{ES}$ treated $=1.88$; ES untreated $=2.54)$. No $/ \mathrm{s} /$ SMT generalization was evidenced during treatment of /d/ or / $\mathrm{t} /$ SMTs. Generalization was evidenced on 2 of 10 $/ \mathrm{s} /$ SMTs during treatment of the $/ \mathrm{g} / \mathrm{SMTs}$ (ES treated = 0.51; ES untreated $=0.61$ ).

Generalization of the treated /d/ SMTs that differed substantially in phonetic structure from those treated, occurred on $20 \%$ for word final $/ \mathrm{d} /(\mathrm{ES}=3.1), 20 \%$ for word initial $/ \mathrm{t} /(\mathrm{ES}=0.83), 50 \%$ for word initial $/ \mathrm{k} /(\mathrm{ES}=$ $0.92)$ and $50 \%$ for word final $/ z /(E S=0.69)$. Generalization to those same untreated SMTs for the treated $/ \mathrm{t} / /$ was $20 \%$ for word final /d/ (ES $=0.70), 10 \%$ for word initial $/ \mathrm{t} / /(\mathrm{ES}=2.5), 0 \%$ for word initial $/ \mathrm{k} /(\mathrm{ES}=0.41)$ and $30 \%$ for word final $/ \mathrm{z} /(\mathrm{ES}=1.59)$. Treatment of the $/ \mathrm{g} / \mathrm{SMTs}$ generalized $0 \%$ for word final $/ \mathrm{d} /(\mathrm{ES}=0.6), 30 \%$ for word initial $/ \mathrm{t} /(\mathrm{ES}=2.19), 50 \%$ for word initial $/ \mathrm{k} /(\mathrm{ES}=1.84)$ and $10 \%$ for word final $/ z /(E S=2.48)$. Treatment of the /s/ initial SMTs evidenced no generalization to any of these SMTs.

Maintenance of the treated STMs was evidenced for 5 of the $5 / \mathrm{d} /$ initial SMTs (4 met criterion; ES $=1.33$ ), 5 of the 5 for the $/ \mathrm{t} \mathrm{f} /$ initial SMTs (1 met criterion; ES = 3.5), for 5 of $5 / \mathrm{g} /$ initial SMTs (all 5 met criterion; ES = 9.02) and 4 of 5 for the /s/ initial SMTs (1 met criterion; ES = 0.94). Maintenance of treated /d/ initial SMTs to untreated /d/ initial SMTs was evidenced for 2 of 5 SMTs (1 met criterion; ES = 0.81); treated $/ \mathrm{t} \int /$ initial SMTs to untreated $/ \mathrm{t} / /$ initial SMTs was 5 of 5 ( 2 met criterion; ES $=4.29$ ); treated $/ \mathrm{g} /$ initial SMTs to untreated $/ \mathrm{g} /$ initial SMTs was 5 of 5 (all met criterion; ES $=12.17$ ) and treated $/ \mathrm{s} /$ SMTs to untreated /s/ initial SMTs was 3 of 5 (all met criterion; $\mathrm{ES}=0.73$ ).

Maintenance to the untreated SMTs that differed substantially in phonetic structure was 3 of 10 (all to criterion; $\mathrm{ES}=0.65)$ for the $/ \mathrm{d} /$ final SMTs, 6 of 10 (2 to criterion; $\mathrm{ES}=1.17)$ for $/ \mathrm{J} /, 8$ of 10 (6 to criterion; $\mathrm{ES}=2.93$ ) for $/ \mathrm{k} /$ and 7 of 10 (3 to criterion; ES = 1.56) for $/ \mathrm{z} /$. These maintenance effects are difficult to attribute to the intervention on any individual SMTs as they may be the result of both treatment and generalization effects across targets.

Treatment for P2 was administered on only 2 sets of SMTs. They yielded positive acquisition effects for 5 of the $5 / \mathrm{g} /$ initial SMTs (4 met criterion; ES $=1.8$ ) and on 5 of the $5 / \mathrm{n} /$ final SMTs (3 met criterion; ES $=0.56$ ). Generalization to similar SMTs (/g/ initial SMTs) with/g/ ini- tial SMTs treatment occurred for 4 of the 5 SMTs and 2 met criterion $(\mathrm{ES}=1.37)$. Generalization was also evident for 4 of 5 SMTs for /n/ initial treatment (all 4 met criterion; ES = 1.47). Generalization from the treated $/ \mathrm{g} / \mathrm{SMTs}$ to untreated SMTs that differed substantially in phonetic structure occurred on $20 \%$ for $/ \mathrm{n} /$ final $(\mathrm{ES}=0.6), 89 \%$ for $/ \mathrm{k} /$ initial ( $45 \%$ to criterion; $\mathrm{ES}=2.37,10 \%$ for $/ \mathrm{d} /$ final $(\mathrm{ES}=0.4), 10 \%$ to criterion for $/ \mathrm{n} / \mathrm{initial}(\mathrm{ES}=0.28)$ and $10 \%$ to $/ \mathrm{z} /$ final $(\mathrm{ES}=-0.5)$. No generalization was realized for $/ \mathrm{s} /$ initial and / $\mathrm{t} /$ final SMTs. Generalization from the treated $/ \mathrm{n} /$ final SMTs to the untreated SMTs that differed substantially in phonetic structure occurred on $20 \%$ for $/ \mathrm{n} /$ initial $(\mathrm{ES}=1.7), 20 \%$ to criterion for $/ \mathrm{t} /$ final $(\mathrm{ES}=0.1)$ and $10 \%$ to $/ \mathrm{z} / \mathrm{final}(\mathrm{ES}=-0.16)$. No generalization was realized for $/ \mathrm{k} /$ initial, $/ \mathrm{s} /$ initial, $/ \mathrm{d} /$ final and $/ \mathrm{k}$ / final SMTs. Maintenance was not assessed for P2.

Between 7 and 12 sessions were required to reach criterion for P1 under the $100 \%$ feedback condition, which was administered first. Three to 6 sessions were required under the $50 \%$ feedback condition. The $50 \%$ condition was administered first for P2 and 27 sessions were required to reach criterion. The $100 \%$ condition required 23 sessions to reach criterion.

\section{Summary and Conclusions}

The data illustrated in figure 1 provide a reasonable representation of the overall results for the 2 participants with this online AVKF plus clinician-supplied feedback treatment. While this must be regarded as a phase-II clinical study, we interpret these results as firm support for the attribution of the behavioral changes realized to the intervention. This is based on appropriate baselines, control conditions, along with changes that occurred in appropriate temporal relationships to the intervention. The kinematic plus clinician-supplied feedback about the realized perceptual accuracy resulted in improvement to criterion for nearly all exemplars of the targeted SMTs. There was also substantial generalization to similar and dissimilar SMTs and maintenance of the gains from both acquisition and generalization. Convergent findings from both the visual-perceptual analyses and effect size calculations support this conclusion. The number of SMTs that were judged to improve above baseline levels with AVKF plus clinician-supplied feedback was nearly $100 \%$ for both participants, and the computed effect sizes were in general agreement with these visual inspection results. The effect sizes did however vary considerably across treated targets; averaged 3.28 and ranged from 
1.05 to 7.17 for $\mathrm{P} 1$, and averaged 1.18 and ranged from 0.56 to 1.80 for P2. Generalization to SMTs with similar phonetic structure yielded effect sizes that ranged from 0.45 to 6.08 (average $=2.07$ ) for $\mathrm{P} 1$ and from 1.37 to 1.47 (average $=1.42$ ) for P2. Generalization to untreated SMTS that differed substantively from the treated targets yield effect sizes that ranged from 0.41 to 3.10 (average $=1.24$ ) for P1 and from -0.5 to 2.37 (average positive ES $=1.07$ ) for P2. P1 also evidenced maintenance of treated SMTs that ranged from 0.94 to 9.02 (average $=3.45$ ) and generalization gains across similar SMTs ranged from 0.73 to 12.17 (average 4.50). Generalization to dissimilar SMTs ranged from 0.65 to 2.93 (average $=1.57$ ) for P1.

The fact that generalization to untreated probes occurred across disparate phonetic configurations including place, manner, voicing features and vowel advancement (back, mid, front) and height (high, mid, low) is difficult to interpret based on notions of distinctive feature representations as an organizing principle for determining response class and for selecting SMTs for treatment. Additional analyses of these and similar data from many more participants will be used to explore other organizing principles for selecting treatment targets for speech movement disorders.

The effects of feedback frequency were confounded by limited patterns of randomization and thus are uninterpretable for the individuals in this study. Another limitation of this study is the restricted sampling opportunity for some SMT generalization probes, due to the length of a treatment phase. This study also did not disambiguate the contributions of knowledge of performance, knowledge of results and clinician-supplied feedback on the treatment effects. Future investigations will examine the temporal parameters of feedback (delayed vs. immediate), practice variables (random vs. blocked) and other feedback variables (i.e. summary vs. immediate) that have been identified in the motor learning literature, in order to assist in developing more efficacious and effective treatments for AOS.

This study represents an early attempt to explore the effects of augmented movement feedback on the successful acquisition, generalization and maintenance of speech in AOS. It was not designed to assess the overall efficacy of the intervention. Its efficacy and effectiveness for communication might profitably be studied once positive behavior change attributable to this intervention is firmly established with multiple replications on a sizable population with AOS.

\section{Acknowledgements}

This research was supported by the Department of Veterans Affairs, Veterans Health Administration, Rehabilitation Research and Development Service: Award \#B3670R to Malcolm R. McNeil.

\section{References}

1 Van der Merwe A: A theoretical framework for the characterization of pathological speech sensorimotor control; in McNeil MR (ed): Clinical Management of Sensorimotor Speech Disorders. New York, Thieme, 2008, pp 3-18.

2 McNeil MR, Robin DA, Schmidt RA: Apraxia of speech: definition, differentiation and treatment; in McNeil MR (ed): Clinical Management of Sensorimotor Speech Disorders. New York, Thieme, 1997, pp 311-344.

$\checkmark 3$ Ziegler W: Psycholinguistic and motor theories of apraxia of speech. Semin Speech Lang 2002;23:231-244

-4 Maas E, Robin DA, Austermann Hula SN, Freedman SE, Wulf G, Ballard KJ, Schmidt RA: Principles of motor learning in treatment of motor speech disorders. Am J Speech Lang Path 2008;17:277-298.

5 Wambaugh JL, Duffy JR, McNeil MR, Robin DA, Rogers MA: Evidence-based guidelines for the management of acquired apraxia of speech: treatment descriptions and recommendations. J Med Speech Lang Pathol 2006; 14:xv-Xxxii.
6 Schmidt RA, Lee TD: Motor Control and Learning: A Behavioral Emphasis. Champaign, Human Kinetics, 2005.

7 Schmidt RA, Lee TD: Motor Control and Learning: A Behavioral Emphasis. Champaign, Human Kinetics, 2005.

8 Park JH, Shea CH, Wright DL: Reduced-frequency concurrent and terminal feedback: a test of the guidance hypothesis. J Mot Behav 2007;32:287-296.

-9 Winstein C, Schmidt R: Reduced frequency of knowledge of results enhances motor skill learning. Res Q Exerc Sport 1999;70: 33-40.

10 Katz WF, Bharadwaj SV, Carstens B: Electromagnetic articulography treatment for an adult with Broca's aphasia and apraxia of speech. J Speech Lang Hear Res 1999;42: 1355-1366.

11 Knock TR, Ballard KJ, Robin DA, Schmidt RA: Influence of order of stimulus presentation on speech motor learning: a principled approach to treatment for apraxia of speech. Aphasiology 2000;14:653-668.
12 Wambaugh JL, Duffy JR, McNeil MR, Robin DA, Rogers M: Treatment guidelines for acquired apraxia of speech: a synthesis and evaluation of the evidence. J Med Speech Lang Pathol 2006; 14:xv-xxxiii.

13 Dabul B: Apraxia Battery for Adults - 2. Tigard, C.C. Publications, 2000.

14 Goodglass H, Kaplan E, Barresi B: The assessment of aphasia and related disorders, ed 3. Philadelphia, Lippincott Williams \& Wilkins, 2001.

15 Raven JC: The Coloured Progressive Matrices. New York, Psychological Corporation, 1965.

16 McNeil MR, Sung JE, Yang D, Pratt SR, Fossett TR, Pavelko S, Doyle PJ: Comparing connected language elicitation procedures in person with aphasia: concurrent validation of the story retell procedure. Aphasiology 2007;21:775-790. 